\title{
Histomorfometria do corno uterino de gatas (Felis catus) submetidas à ovariosalpingohisterectomia
}

\author{
Histomorphometry of the uterine horn of queens (Felis catus) uterus submitted to \\ ovaryhisterectomy
}

\section{Cristina Maria Rodrigues MONTEIRO ${ }^{1}$; Silvia Helena Venturoli PERRI ${ }^{1}$; Roberto Gameiro de CARVALHO $^{1}$; Adelina Maria da SILVA ${ }^{2}$; Marion Burkhardt KOIVISTO ${ }^{2}$}

\author{
${ }^{1}$ Departamento de Apoio, Produção e Saúde Animal do Curso de Medicina Veterinária da Faculdade de Medicina Veterinária da \\ Universidade Estadual Paulista, Araçatuba-SP, Brasil \\ ${ }^{2}$ Departamento de Clínica, Cirurgia e Reprodução Animal da Faculdade de Medicina Veterinária daUniversidade Estadual \\ Paulista, Araçatuba-SP, Brasil
}

\begin{abstract}
Resumo
Dados histomorfométricos foram obtidos de cornos uterinos de gatas nulíparas $(\mathrm{n}=6)$, primíparas $(\mathrm{n}=6)$, multíparas $(n=6)$ e de gatas tratadas com contraceptivo $(n=6)$.O material coletado após a cirurgia foi fixado em paraformaldeído e incluído em resina Paraplast ${ }^{\oplus}$ para se proceder a microtomia. Os cortes adquiridos foram submetidos à coloração rotineira com hematoxilina e eosina e examinados ao microscópio de luz para a realização das seguintes mensurações:espessura total $(\mu \mathrm{m})$ da parede uterina, endométrio total, diâmetro das glândulas endometriais e altura dos respectivos epitélios glandulares, espessura total do miométrio, do miométrio interno,miométrio externo e estrato vascular.Concluiu-se que: uso de anticoncepcionais e inúmeras gestações alteraram a estrutura uterina; uma única gestação parece não afetar significativamente as estruturas da parede uterina como ocorre nas gatas multíparas; não houve variação significativa das estruturas entre as gatas nulíparas e primíparas, com exceção para miométrio interno; o miométrio total e o endométrio apresentaram variações semelhantes, com exceção para o grupo das gatas tratadas; o miométrio externo apresentou alterações mais marcantes nas gatas tratadas; as variações morfológicas menos marcantes foram relativas ao diâmetro e epitélio glandulares; a presença de glândulas endometriais dilatadas foi encontrada somente nas gatas tratadas.
\end{abstract}

Palavras-chave: Útero. Número de partos. Anticoncepcionais. Felinos.

\begin{abstract}
Histomorphometric data were obtained from cats uterine horns: either nulliparous $(\mathrm{n}=6)$, primiparous $(\mathrm{n}=$ $6)$, multiparous $(n=6)$ and treated with contraceptive $(n=6)$. The material was collected after surgery, fixed in paraformaldehyde and embedded in paraplast ${ }^{\dagger}$ resin to be sliced in a microtome. The obtained sections were stained with hematoxylin - eosin and measured under a light microscope: uterine wall total thickness $(\mu \mathrm{m})$, endometrium total height, endometrial glands diameter and glandular epithelia height, total myometrium, internal and external myometrium and vascular layer thickness. It was concluded that: 1 - contraceptive use and number of pregnancies altered the uterine structure, 2 - one pregnancy does not appear to affect the uterine lining structures as occurs in multiparous cats, 3 - there was no variation on the evaluated structures between nulliparous and primiparous cats except for inner myometrium, 4 - the total myometrium hight and the endometrium hight showed similar variations except for the contraceptive treated cats group, 5 - the outer myometrium showed marked changes in the treated cats, 6 - the less marked morphological variations were for the endometrial glands diameter and glandular epithelium hight, 7 - the presence of dilated endometrial glands was found only in treated cats.
\end{abstract}

Keywords: Uterus. Births number. Contraceptives. felines.

\section{Introdução}

As fêmeas domésticas, em especial as carnívoras, apresentam a estrutura histológica dos órgãos reprodutores dividida em três túnicas: endométrio revestido por epitélio simples colunar e lâmina própria com glândulas tubulares; miométrio constituído por duas porções, a interna circular e a externa longitudinal formada por músculo liso; e a serosa de tecido
Correspondência para:

Cristina Maria Rodrigues Monteiro

Departamento de Apoio, Produção e Saúde Animal, Curso de Medicina

Veterinária, Faculdade de Medicina Veterinária

Universidade Estadual Paulista (UNESP), Campus de Araçatuba

Rua Clóvis Pestana, 793

CEP.: 16050-680, Araçatuba, SP

Tel.: +551836361377

e-mail: monteiro@fmva.unesp.br

Recebido: 22/08/2011

Aprovado: $27 / 06 / 2012$ 
conjuntivo frouxo e mesotélio ${ }^{1,2,3}$. Devido à maior relevância, os estudos sobre as consequências do uso de contraceptivos são mais extensos, como pode ser verificado nas investigações de $\mathrm{Cox}^{4}$, Nelson e Kelly ${ }^{5}$, Sokolowski e VanRavenswaay ${ }^{6}$, Kooistra et al. ${ }^{7}$, Perez et al. ${ }^{8}$ e Murakoshi, Tagawz e Ikeda ${ }^{9}$ e Chatdarong et al. ${ }^{10}$ que reportaram a hiperplasia endometrial cística como a principal ocorrência.

Masculinização de fetos fêmeas, tumores das glândulas mamárias, atraso no parto, aumento da secreção de prolactina e gonadotropinas, diabetes mellitus, acromegalia, supressão da região cortical da adrenal, dermatopatias e distúrbios comportamentais também são alterações citadas por Romagnoli e Concannon ${ }^{11}$ como consequência do uso de progestágenos.

Como os estudos histomorfométricos comparativos entre gatas tratadas com anticoncepcional e aquelas não submetidas a este tratamento são escassos, o objetivo desta pesquisa foi realizar uma avaliação histomorfométrica detalhada comparativa do corno uterino de gatas nulíparas, primíparas, multíparas, comparando-se com gatastratadas com contraceptivo, independentemente do número de parições, para se averiguar com dados numéricos as variações que possam ocorrer nesse órgão.

\section{Material e Método}

Foram utilizados 24 cornos uterinos, sem preferência de lado, provenientes de 24 gatas, sem raça definida, com idades variando entre 5 meses a 10 anos, submetidas à ovariosalpingohisterectomia no Hospital Veterinário do Curso de Medicina Veterinária, Unesp, Campus Araçatuba. Os animais foram distribuídos em quatro grupos: nulíparas $(n=6)$, primíparas $(n=6)$, multíparas $(n=6)$ e tratadas com contraceptivo $(\mathrm{n}=6)$ independentemente do número de parições. $\mathrm{O}$ material coletado, após a cirurgia, foi fixado em paraformaldeído tamponado a $4 \%$, por 24 horas, a $5^{\circ} \mathrm{C}$. Posteriormente, fragmentos com $3 \mathrm{~mm}$ de espessura foram recortados na porção média do corno uterino, processados e incluídos em resina paraplast para se proceder a microtomia. Os cortes adquiridos com aproximadamente $5 \mu \mathrm{m}$ de espessura foram submetidos à coloração rotineira com hematoxilina e eosina e examinados ao microscópio de luz para observação histológica e realização das seguintes mensurações: 1) Espessura total $(\mu \mathrm{m})$ da parede uterina, 2) Endométrio: 2a. Espessura total (cinco campos diferentes em cada amostra histológica), 2b. Diâmetro das glândulas endometriais (10 glândulas em cada amostra histológica para as regulares e dilatadas, quando houver), 2c. Altura do epitélio glandular, 3) Espessura total do miométrio, 3a. Espessurado miométrio interno, 3b. Espessura do miométrio externo e 3c. Espessura do estrato vascular (cinco campos diferentes em cada amostra histológica). As mensurações foram obtidas com o programa Image J (software do National Institute of Mental Health, USA). A estatística constituiuse de análise de variância e as médias foram comparadas pelo teste de Duncan e teste t pareado. O nível de significância adotado foi de $5 \%$.

\section{Resultados e Discussão}

A mensuração da parede uterina de gatas nulíparas revelou espessura delgada (Figura 1) com média de $806,16 \mu \mathrm{m}$, enquanto que nas gatas primíparas (Figura 2), multíparas (Figura 3) e tratadas com contraceptivos (Figura 4), as médias foram $1.180,12 \mu \mathrm{m}$, $1.559,03 \mu \mathrm{m}$ e $2.009,53 \mu \mathrm{m}$, respectivamente. A espessura da parede uterina das gatas nulíparas foi menor que a espessura da parede uterina das gatas primíparas, multíparas e tratadas, porém, em relação às primíparas não apresentou diferença significativa e estas com as multíparas também não apresentou diferença significativa, porém, as tratadas e multíparas foram significativas em relação às nulíparas (Tabela 1). Esses resultados numéricos confirmam que inúmeras gestações e o uso de anticoncepcionais afetam a parede uterina em sua totalidade. 


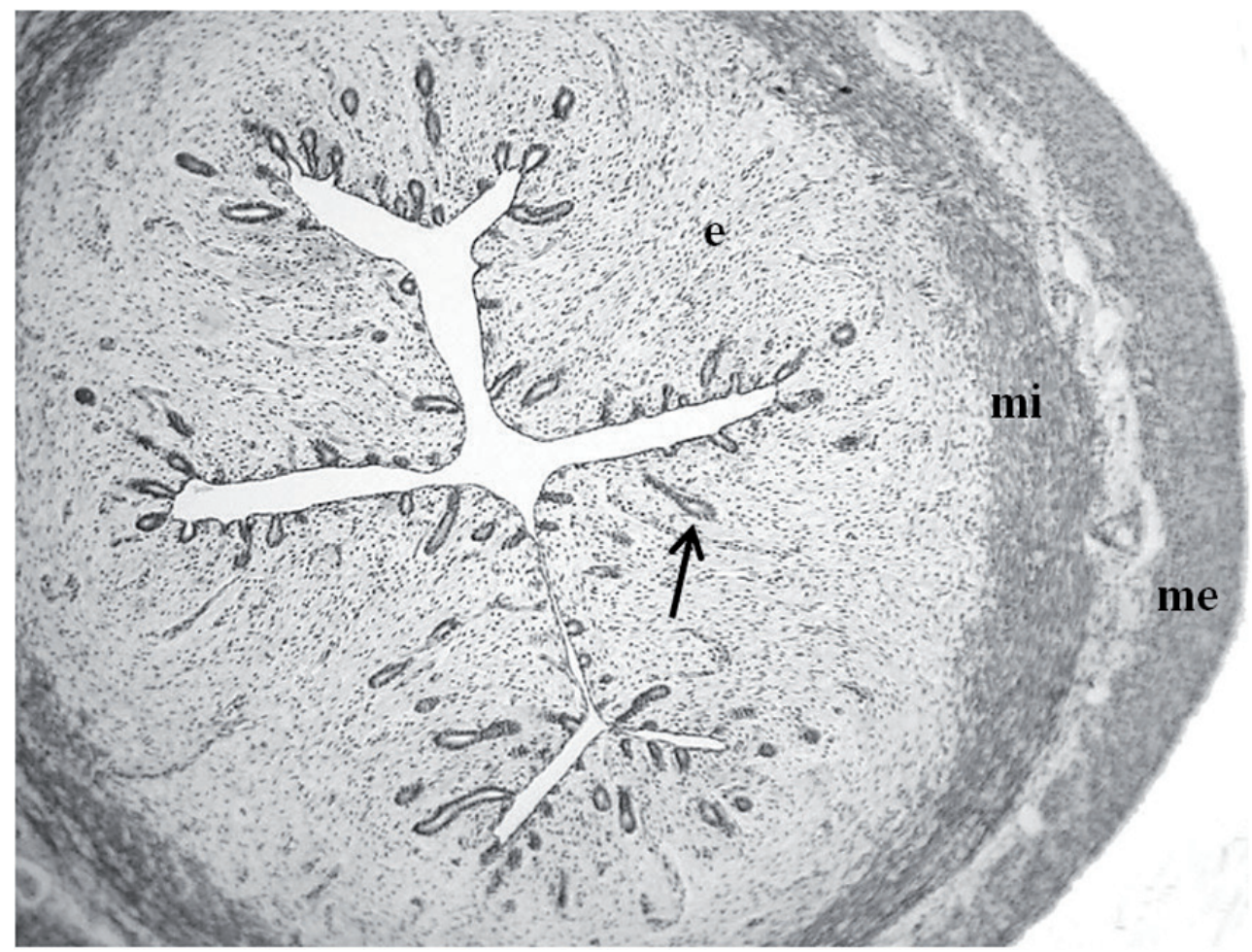

Figura 1 - Fotomicrografia de corno uterino de gata nulípara. Endométrio (e). Glândulas endometriais. (seta). Miométrio interno (mi). Miométrio externo (me). Hematoxilina e Eosina. 4X

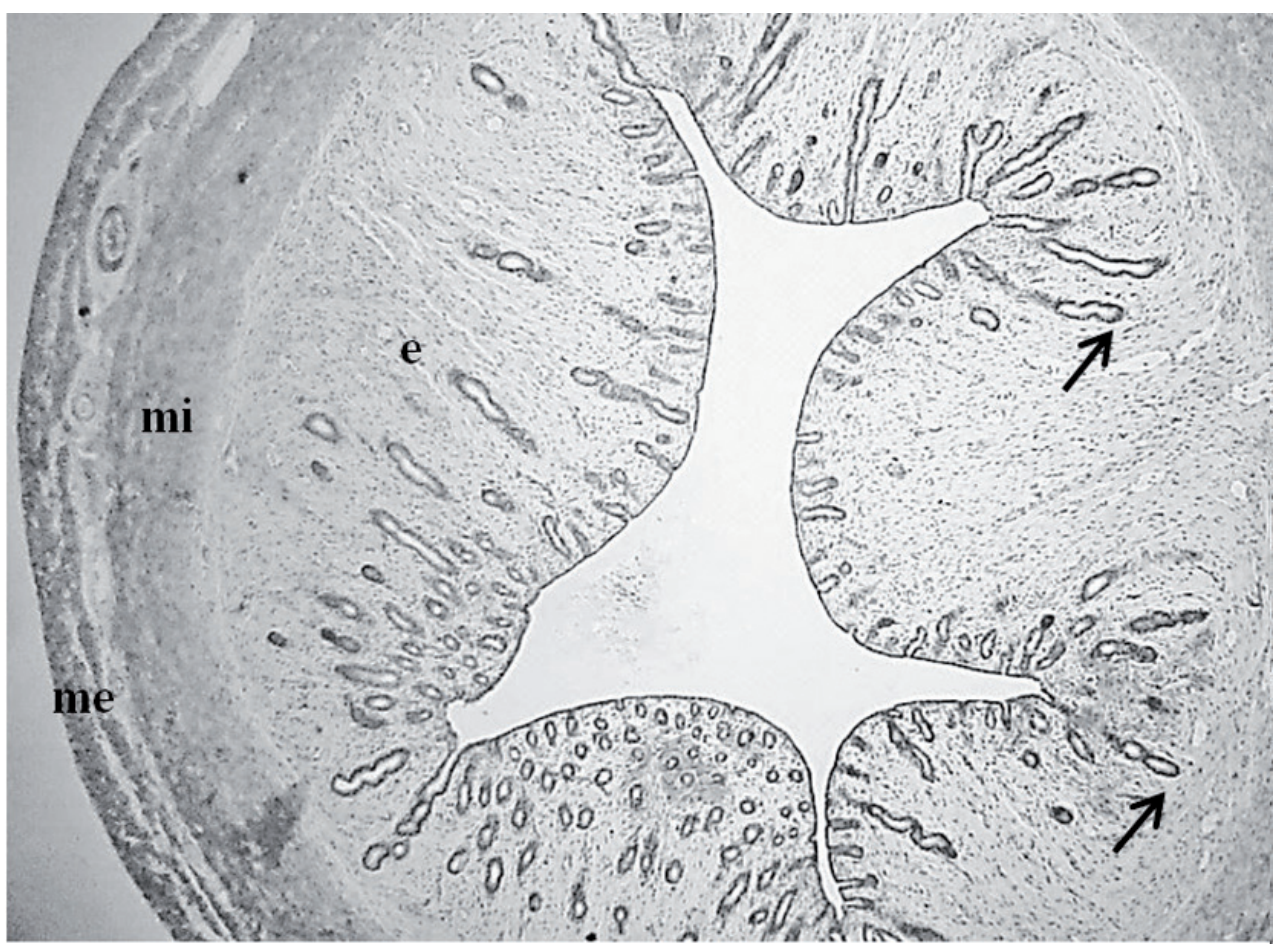

Figura 2 - Fotomicrografia de corno uterino de gata primípara. Endométrio (e). Glândulas endometriais. (setas). Miométrio interno (mi). Miométrio externo (me). Hematoxilina e Eosina. 4X 


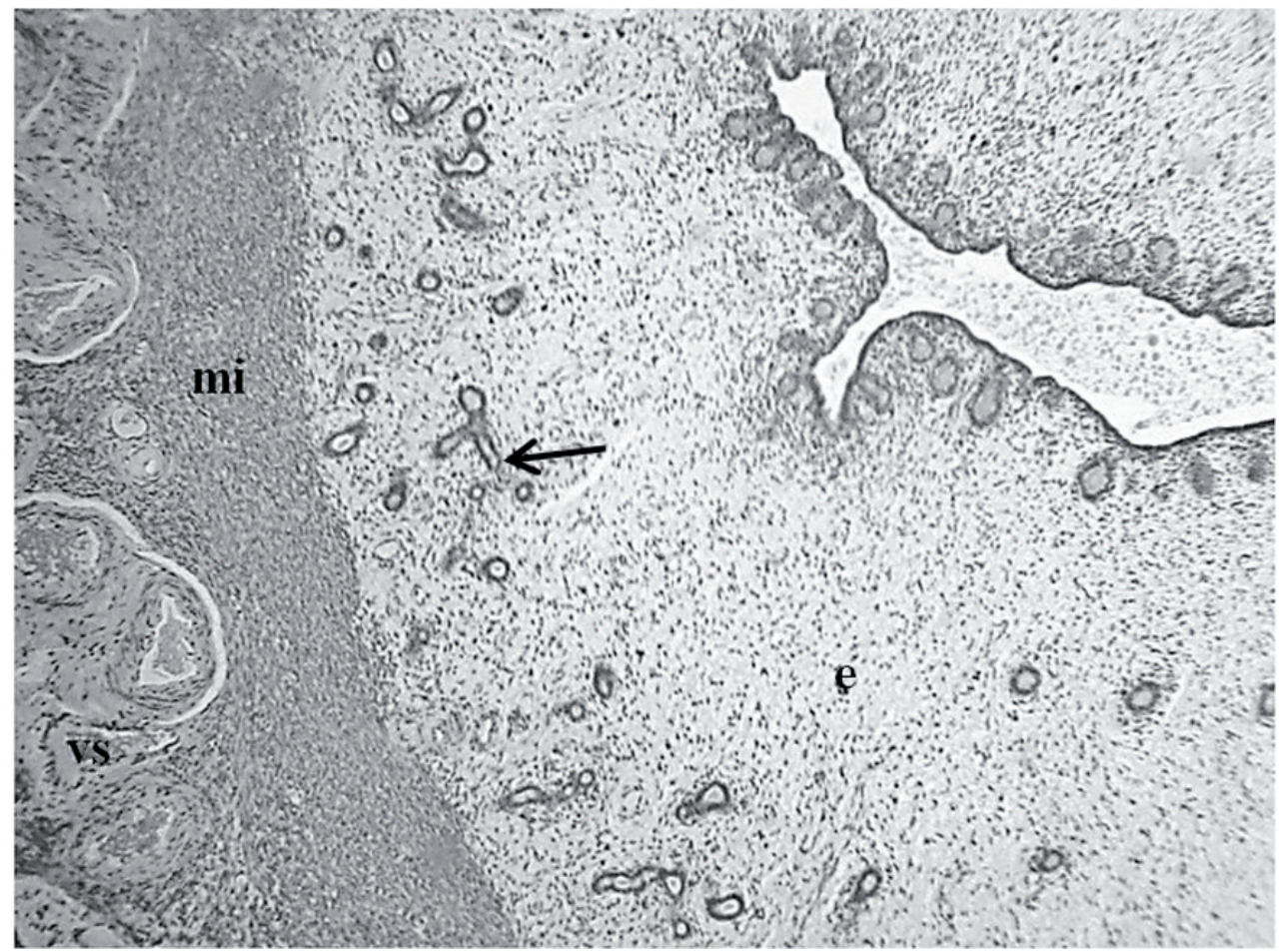

Figura 3 - Fotomicrografia de corno uterino de gata multípara. Endométrio (e). Glândulas endometriais. (seta). Miométrio interno (mi). Estrato vascular com vasos sanguíneos evidentes (vs). Hematoxilina e Eosina. 10X

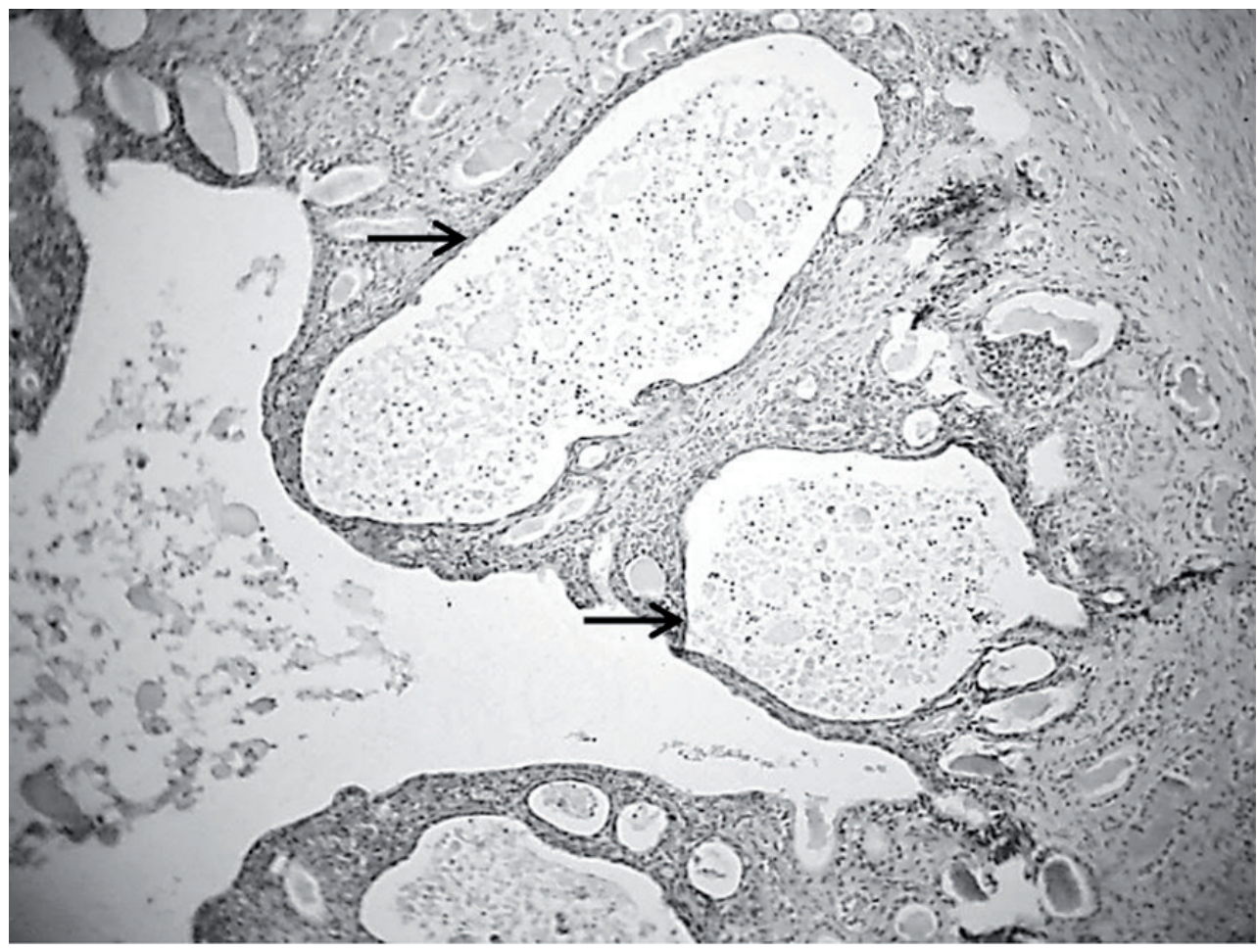

Figura 4 - Fotomicrografia de corno uterino de gata tratada com anticoncepcional. Glândulas endometriais dilatadas (setas). Hematoxilina e Eosina. 4X 
O miométrio total das gatas nulíparas apresentou espessura de $313,75 \mu \mathrm{m}$, menor em relação aos demais grupos, porém, sem diferença significativa com as gatas primíparas, que apresentaram espessura média de 599,27 $\mu \mathrm{m}$. Estas últimas também não apresentaram diferença significativa com o grupo das gatas multíparas cuja média foi de 793,64 $\mu \mathrm{m}$, porém, significativa em relação às nulíparas (Tabela 1 ). De acordo com Augsburger e Kurzi ${ }^{12}$, em cadelas Beagles multíparas houve um aumento de tecido conjuntivo miometrial, fato que pode explicar o aumento da espessura desta camada em animais multíparos. No entanto, a média do miométrio total das gatas tratadas mostrou diferença significativa em relação às demais, em um total de 1392,64 $\mu \mathrm{m}$ (Tabela 1). As conclusões de Chatdarong et al. ${ }^{10}$ corroboram nossos resultados, pois os autores encontraram em gatas tratadas com contraceptivo, miométrio significativamente mais espesso.

Para a subcamada interna do miométrio houve diferença significativa das médias ao se comparar as gatas nulíparas $(151,33 \mu \mathrm{m})$ com os outros grupos, e entre estes, não houve diferença significativa com as respectivas espessuras de 265,25 $\mu \mathrm{m}, 325,95$ $\mu \mathrm{m}$ e $356,10 \mu \mathrm{m}$ para gatas primíparas, multíparas e tratadas. A subcamada externa não mostrou diferença significativa entre as médias dos grupos das nulíparas, primíparas e multíparas $(110,09 \mu \mathrm{m}$, $170,57 \mu \mathrm{m}$ e $218,37 \mu \mathrm{m}$, respectivamente), porém, apresentou diferença significativa com o grupo das tratadas, cuja média foi $709,23 \mu \mathrm{m}$. O estrato vascular não apresentou diferença estatisticamente significativa entre o grupo das gatas nulíparas $(93,75 \mu \mathrm{m})$ e primíparas $(180,73 \mu \mathrm{m})$, mas houve diferença em comparação às multíparas e tratadas com 306,74 e 330,13 $\mu \mathrm{m}$, respectivamente (Tabela 1). Augsburger e Kurzi ${ }^{12}$ citaram em cadelas multíparas a presença de vasos sanguíneos com espessamento da túnica média em artérias e da adventícia em veias, o que leva consequentemente a um aumento do estrato vascular.

Do ponto de vista histomorfométrico, as variações mais evidentes ocorreram no miométrio total e na subcamada miometrial externa ao se comparar animais tratados com os demais e, com exceção para o miométrio interno, todas as médias aferidas aos animais nulíparos e primíparos não apresentaram diferença significativa, fato esperado, pois uma única gestação parece não afetar significativamente as estruturas da parede uterina como ocorre nas gatas multíparas.

A espessura total do endométrio entre as gatas primíparas $(653,49 \mu \mathrm{m})$, multíparas $(819,00 \mu \mathrm{m})$ e tratadas $(735,92 \mu \mathrm{m})$ não apresentou diferença estatisticamente significativa entre si, porém, houve diferença estatisticamente significativa entre as gatas multíparas e nulíparas $(473,45 \mu \mathrm{m})$ e estas não apresentaram diferença com as primíparas e tratadas (Tabela 1). As médias do diâmetro das glândulas endometriais re-

Tabela 1- Média e erro padrão da média das variáveis estudadas nos cornos uterinos de gatas nulíparas (N) (n=6), primíparas $(P)(n=6)$, multíparas $(M)(n=6)$ e tratadas com contraceptivo $(T)(n=6)$ - Araçatuba (SP) - 2011

\begin{tabular}{|c|c|c|c|c|c|c|c|c|}
\hline \multirow{2}{*}{ G } & \multirow{2}{*}{$\begin{array}{l}\text { Parede uterina } \\
\qquad(\mu \mathrm{m})\end{array}$} & \multicolumn{4}{|c|}{ Miométrio ( $\mu \mathrm{m})$} & \multicolumn{3}{|c|}{ Endométrio $\mu \mathrm{m}$ ) } \\
\hline & & Total & Interno & Externo & Estrato vascular & Total & Glândulas & Epitélio \\
\hline $\mathrm{P}$ & $1180,12 \pm 127,14 b c$ & $599,27 \pm 139,52 \mathrm{bc}$ & $265,25 \pm 39,17 \mathrm{a}$ & $170,57 \pm 34,64 b$ & $180,73 \pm 44,47 \mathrm{bc}$ & $653,49 \pm 45,04 \mathrm{ab}$ & $27,52 \pm 0,91 b$ & $8,66 \pm 0,22 \mathrm{a}$ \\
\hline M & $1559,03 \pm 185,39 a b$ & $793,64 \pm 72,56 b$ & $325,95 \pm 33,65 a$ & $218,37 \pm 28,63 b$ & $306,74 \pm 26,79 a b$ & $819,00 \pm 169,92 \mathrm{a}$ & $33,76 \pm 4,74 \mathrm{ab}$ & $9,37 \pm 0,75 \mathrm{a}$ \\
\hline $\mathrm{T}$ & $2009,53 \pm 253,58 a$ & $1392,64 \pm 193,35 a$ & $356,10 \pm 25,78 a$ & $709,23 \pm 143,77 a$ & $330,13 \pm 76,80 a$ & $735,92 \pm 99,59 \mathrm{ab}$ & $39,31 \pm 2,86 \mathrm{a}$ & $10,03 \pm 1,04 \mathrm{a}$ \\
\hline
\end{tabular}

Médias seguidas de letras distintas, na coluna, diferem entre si pelo teste de Duncan $(\mathrm{p}<0,05)$ 
gulares não mostraram diferença significativa entre as gatas nulíparas, primíparas e multíparas, com as seguintes mensurações de $26,18 \mu \mathrm{m}, 27,52 \mu \mathrm{m}$ e 33,76 $\mu \mathrm{m}$, respectivamente, porém, houve diferença significativa entre as gatas tratadas $(39,31 \mu \mathrm{m})$ e nulíparas e entre gatas tratadas e primíparas. As alturas dos epitélios não mostraram médias com diferenças significativas entre os grupos estudados (Tabela 1). Esses últimos achados sugerem poucas alterações morfológicas relativas às glândulas endometriais regulares, porém, não ocorreu o mesmo com a parede endometrial em seu total onde se verificou maior espessura nas gatas multíparas e não nas tratadas, pois nessas últimas, as variações mais distintas ocorreram nas glândulas endometriais que estavam dilatadas.

Estas alterações se fizeram presentes como glândulas císticas, confirmado pelas mensurações com diâmetro médio de $185,50 \mu \mathrm{m}$, significativo quando comparado às glândulas regulares, cujo diâmetro médio foi de 39,31 $\mu \mathrm{m}$, enquanto as médias das alturas dos epitélios foram aproximadas, sem diferença significativa (Tabela 2). Essas alterações confirmadas pelas mensurações efetuadas sugerem ocorrência de hiperplasia endometrial cística, síndrome que se assemelha aos achados de Bigliardi et al. ${ }^{13}$ em cadelas, po- rém, bem menos comum em gatas. Por outro lado, as conclusões de Nelson e Kelly ${ }^{5}$ e Sokolowski e VanRavenswaay $^{6}$, sobre o uso de contraceptivos, mostraram que há o aparecimento de hiperplasia endometrial cística em cadelas, de grau leve com o uso de doses baixas e de grau moderado a severo com o uso de altas dosagens.

\section{Conclusões}

Frente aos dados obtidos, pode-se concluir que: uso de anticoncepcionais e inúmeras gestações alteraram a estrutura uterina; uma única gestação parece não afetar significativamente as estruturas da parede uterina como ocorre nas gatas multíparas; não houve variação significativa das estruturas entre as gatas nulíparas e primíparas, com exceção para miométrio interno; o miométrio total e o endométrio apresentaram variações semelhantes, com exceção para o grupo das gatas tratadas; o miométrio externo apresentou alterações mais marcantes nas gatas tratadas; as variações morfológicas menos marcantes foram relativas ao diâmetro e altura do epitélio das glândulas regulares e as glândulas endometriais dilatadas foram encontradas somente nas gatas tratadas.

Tabela 2 - Média e erro padrão da média do diâmetro e da altura do epitélio das glândulas normais e dilatadas dos cornos uterinos de gatas tratadas com contraceptivo - Araçatuba (SP) - 2011

\begin{tabular}{ccc}
\hline Glândulas & Diâmetro $(\boldsymbol{\mu m})$ & Altura do Epitélio $(\boldsymbol{\mu m})$ \\
\hline Normais & $39,31 \pm 2,86 \mathrm{~b}$ & $10,03 \pm 1,04 \mathrm{a}$ \\
Dilatadas & $185,50 \pm 28,77 \mathrm{a}$ & $8,77 \pm 0,65 \mathrm{a}$ \\
\hline
\end{tabular}

Médias seguidas de letras distintas, na coluna, diferem entre si pelo teste t pareado $(\mathrm{p}<0,05)$

\section{Referências}

1.BANKS, W. J. Histologia veterinária aplicada. 2. ed. São Paulo: Manole, 1992. 629 p.

2. DELLMANN, H. D. Histologia veterinária. 2. ed. Zaragoza: Acribia, 1993. 398 p.

3.HAFEZ, E. S. E.; HAFEZ, B. Reprodução animal. 7. ed. São Paulo: Manole, 2004. 513 p.

4. COX, J. Progestagens in bitches. A review. Journal Small Animal Practice, v. 11, n. 12, p. 759, 1970.
5. NELSON, L. W.; KELLY, W. A. Progestogen-related gross and microscopic changes in female Beagles. Veterinary Pathology, v. 13, n. 2, p. 143-56, 1976.

6. SOKOLOWSKI, J. H.; VANRAVENSWAAY, F. Effects of melengestrol acetate on reproduction in the Beagle bitch. American Journal of Veterinary Research, v. 8, n. 37, p. 943 945, 1976.

7.KOOISTRA, H. S.; OKKENS, A. C.; MOL, J. A.; VAN GARDEREN, E.; KIPENSTEIJN, J.; RIJNBERK, A. Lack 
of association of progestin-induced cystic endometrial hyperplasia with $\mathrm{GH}$ gene expression in the canine uterus. Journal of Reproduction and Fertility Supplement, v. 51, p. 355-361, 1997

8. PEREZ, J. F.; CONLEY, A. J.; DIETER, J. A.; SANZ, O. J.; LASLEY, B. L. Studies on the origin of ovarian interstitial tissue and the incidence of endometrial hyperplasia in domestic and feral cats. General and Comparative Endocrinology, v. 116, n. 1 , p. 10-20, 1999.

9. MURAKOSHI, M.; TAGAWA,M.;IKEDA, R. Histopathological studies of subcutaneous implantation of chlormadinone acetate (CMA) for preventing estrus in queens. Tokai Journal of Experimental Clinical Medicine, v. 1, n. 25, p. 7-10, 2000.

10.CHATDARONG, K.; RUNGSIPIPAT, A.; AXNÉR, E.; FORSBERG, C. L. Hysterographic appearance and uterine histology at different stages of the reproductive cycle and after progestagen treatment in the domestic cat. Theriogenology, v. 64, n. 1, p. 12-29, 2005

11.ROMAGNOLI, S.; CONCANNON, P. W. Clinical use of progestins in bitches and queens: A review. In: CONCANNON, P. W.; ENGLAND, G.; VERSTEGEN, J.; LINDE-FORSBERG, C. (Ed.). Recent advances in small animal reproduction publisher. New York: Ithaca, 2003. p. 1-17.

12.AUGSBURGER, R. H.; KURZI, M. Histomorphologic and morphometric evaluation of the uterine horns in nulliparous and multiparous beagles. American Journal of Veterinary Research, v. 65, n. 5, p. 552-558, 2004.

13.BIGLIARDI, E.; PARMIGIANI, E.; CAVIRANI, S.; LUPPI A.; BONATI, L.; CORRADI, A. Ultrasonography and cystic hyperplasia-pyometra complex in the bitch. Reproduction in Domestic Animals, v. 39, n. 3, p. 136-140, 2004. 